Ann. Sci. forest., 1967, 24 (4), 259-275.

\title{
ÉTUDE DU RÔLE DU MANGANÈSE DANS LA RÉGÉNÉRATION DE LA SAPINIÈRE VOSGIENNE
}

\author{
G. VALLÉE \\ Station de Recherches sur les Sols forestiers et la Fertilisation, \\ Centre national de Recherches forestières, 54 - Nancy \\ Institut national de la Recherche agronomique
}

SOMMAIRE

Étude du cycle du manganèse dans le mor et le mull des sapinières des Vosges.

Cette étude conduit à une explication de la toxicité de cet élément pour les jeunes semis de sapin.

\section{PRÉAMBULE}

Pendant plus de deux années, nous avons travaillé à Nancy au problème de la régénération du sapin dans la forêt vosgienne.

Ces travaux dirigés par Monsieur le Professeur Duchaufour (Centre de Pédologie du C.N.R.S.), Monsieur le Professeur Mangenot (Faculté des Sciences), Monsieur Dommergues (Centre de Pédologie du C.N.R.S.) et Monsieur Bonneau (Centre national de Recherches forestières) ont été exposés en détail dans une thèse de Docteur-Ingénieur soutenue en 1966 devant la Faculté des Sciences de Nancy.

Nous en donnons ici les résultats essentiels.

\section{INTRODUCTION}

Depuis longtemps les forestiers ont observé une alternance entre la hêtraie et la sapinière à hêtre (Fagus sylvatica L.) sur les sols à mull des Vosges. Les sapinières à mull après une certaine évolution sont envahies par le hêtre, la régénération de sapin pectiné étant inhibée. Inversement, la vieille hêtraie favorisée par les forestiers dans le passé se voit envahie par le sapin (Abies pectinata D.C.). 
RousSEAU (1960) par des analyses de sols comparatives a démontré que les mull contenaient de fortes concentrations en Mn échangeable. Si celui-ci n'empêchait pas la germination des graines de sapin, il devenait toxique pour les semis par un phénomène physico-chimique :

$1^{0}$ inhibition du développement des racines;

$2^{\circ}$ formation d'une gaine d'oxyde manganique autour des racines des semis par l'alternance de sécheresse et d'humidité.

Quelle est l'origine de l'accumulation du Mn échangeable dans les mull ? Nous nous sommes donnés comme but l'étude de deux points particuliers :

$1^{0}$ est-il possible que par sa physiologie, le sapin concentre le $\mathrm{Mn}$ dans ses aiguilles à partir de la roche-mère et le retourne à l'humus qui l'accumule : d'où étude de la nutrition du sapin suivant les types d'humus et de roches-mères ;

$2^{a}$ si le sapin concentre le Mn dans sa litière, pourquoi n'y a-t-il pas accumulation dans les moder et mor contrairement aux mull ? D'où étude de l'évolution du $\mathrm{Mn}$ des litières suivant les types d'humus de la sapinière.

\section{1. - ASPECT NUTRITIF}

Le manganèse est un élément qui se retrouve en quantité assez importante dans les feuilles des arbres. La teneur est très variable d'une espèce à l'autre et les symptômes de toxicité sont difficiles à déceler.

Le tableau 1 nous donne les teneurs foliaires moyennes en Mn d'arbres adultes (dominants ou codominants) de cinq stations vosgiennes sur trois roches-mères différentes accompagné des teneurs en manganèse échangeable des humus de chacune des stations.

TABLEAU 1

Teneurs foliaires moyennes et variations individuelles en $\mathrm{Mn}$ de 5 stations vosgiennes

\begin{tabular}{|c|c|c|c|c|c|}
\hline \multirow{2}{*}{ Stations } & \multicolumn{2}{|c|}{ Teneurs foliaires } & \multirow{2}{*}{$\begin{array}{c}\text { Mn éch. } \\
\text { m.é/100 g } \\
\text { séché à l'air }\end{array}$} & \multirow{2}{*}{$\begin{array}{l}\mathrm{pH} \text { de } \\
\text { l'humus }\end{array}$} & \multirow{2}{*}{$\begin{array}{c}\text { Semis } 1 \text { à } 5 \\
\text { ans. Mn } \\
\text { foliaire. } \\
\text { Moyenne }\end{array}$} \\
\hline & $\begin{array}{l}\text { Moyenne } \\
\text { Station }\end{array}$ & $\begin{array}{c}\text { Variations } \\
\text { individuelles }\end{array}$ & & & \\
\hline 10 Mull our orès intermó & $\mathrm{ppm}$ & ppm & & & $\mathrm{ppm}$ \\
\hline diaire .................. & 2828 & $1530-3890$ & 0,32 & 4,4 & 2530 \\
\hline 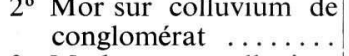 & 291 & $140-440$ & 0,05 & 3,4 & 290 \\
\hline $\begin{array}{l}3^{\circ} \text { Moder sur colluvium } \\
\text { de conglomérat } \ldots . . .\end{array}$ & 531 & $290-750$ & 0,05 & 3.4 & 570 \\
\hline $4^{\circ}$ Mull sur granite....... & 1005 & $700-1450$ & 0,60 & 4,5 & 1760 \\
\hline $5^{\circ}$ Moder sur granite & 167 & $100-260$ & 0,02 & 4,1 & 270 \\
\hline
\end{tabular}




\section{Commentaires}

$1^{\circ}$ Pour un même type d'humus, il existe une différence sensible entre les teneurs en Mn suivant les roches-mères.

$2^{\circ}$ Les teneurs foliaires moyennes en Mn tant pour les adultes que pour les semis sont de beaucoup plus fortes dans les stations à mull, de même que la variation individuelle des teneurs se situe à un niveau plus élevé.

$3^{0}$ Les fortes teneurs en Mn des aiguilles de sapin et des mull appuie la thèse de Rousseau (1960). Cependant, même si le sapin adulte ne semble pas souffrir de cette forte nutrition en $\mathrm{Mn}$, on ne saurait admettre cette remarque pour le semis dont le système radiculaire est confiné à l'humus où la concentration en Mn échangeable est forte.

$4^{\circ}$ Le mull, avec un $\mathrm{pH}$ de 4,5 et dans les conditions climatiques présentes est en équilibre instable et donc sujet aux variations de topographie, d'exposition et même de composition des peuplements forestiers (DUCHAUfOur et al. 1958).

\section{2. - ASPECT DE L'ÉVOLUTION DU Mn DES LITIÈRES, SUIVANT LES TYPES D'HUMUS DE SAPINIÈRE}

Nous aborderons le problème de deux façons :

$1^{\circ}$ par 1'incubation de mull et moder de sapinière enrichis en $\mathrm{MnSO}_{4}, \mathrm{MnO}_{2}$, litière de sapin et de hêtre afin d'étudier le devenir du Mn des litières dans la sapinière.

$2^{\circ}$ par l'établissement d'un bilan partiel du cycle du Mn dans les types de sapinières à mull et à moder.

\section{A. - INCUBATION DE MULL ET MODER DE SAPINIÈRE ENRICHIS}

Deux buts sont poursuivis dans cette expérience :

$1^{\circ}$ d'abord une étude de 1'action de la microflore du mull et moder sur le $\mathrm{Mn}^{++}\left(\mathrm{MnSO}_{4}\right)$ et $\mathrm{Mn}^{4+}\left(\mathrm{MnO}_{2}\right)$, et enfin,

$2^{\circ}$ comparer cette action aux processus d'évolution du Mn des litières incorporées aux humus et voir l'influence possible des litières.

\section{a) Description de l'expérience d'incubation}

\section{Provenance des humus et litières}

Les humus et litières ont été échantillonnés dans la forêt du Ban d'Etival (Vosges) à la fin d'octobre 1964. Le mull ainsi que les litières proviennent d'une sapinière à hêtre (parcelle $n^{0} 3$ ), tandis que le moder provient d'une sapinière pure située 
dans la parcelle $\mathrm{n}^{0} 5$. Les litières ne consistent qu'en des aiguilles de sapin et feuilles de hêtre déjà roussies à cette époque mais non tombées et échantillonnées dans la sapinière à mull pour leur richesse en $\mathrm{Mn}$.

\section{Récipient utilisé}

Boîte en polyéthylène carrée de $10 \times 10 \mathrm{~cm}$ de côté et $6,5 \mathrm{~cm}$ de profondeur avec couvercle.

\section{Poids d'humus par boîte}

L'enrichissement se faisant par unité de volume parce que la distribution de la microflore se fait dans l'espace et non suivant la masse, nous avons mis un volume égal d'humus dans chaque boîte correspondant à $157 \mathrm{~g} \pm 10 \%$ de mull et $68 \mathrm{~g}$ $\pm 10 \%$ de moder tamisé à $2 \mathrm{~mm}$ et séché à $105^{\circ} \mathrm{C}$. L'erreur de $10 \%$ a été évaluée statistiquement car l'incubation a été faite avec les humus préalablement tamisés par un treillis à mailles carrées de $1,5 \mathrm{~cm}^{2}$ de manière à conserver la structure des humus.

\section{L'enrichissement}

$25 \mathrm{~g}$ de litière, $600 \mathrm{mg}$ de $\mathrm{MnSO}_{4}-\mathrm{H}_{2} \mathrm{O}$ et $300 \mathrm{mg}$ de $\mathrm{MnO}_{2}$ ont été incorporés aux humus avec quatre répétitions par traitement. Deux répétitions étaient conservées à environ $120 \%$ d'humidité équivalente $(120 \%$ H.E.) tandis que les deux autres subissaient un régime d'hygropériodisme.

\section{Conditions d'incubation}

Température : $28^{\circ} \mathrm{C}$.

Aération : le couvercle des boîtes des incubations à $120 \%$ H. E. était entrebaillé de sorte à laisser une circulation d'air, tandis que celles subissant 1'hygropériodisme étaient sans couvercle.

Humidité : les échantillons à $120 \% \mathrm{H}$. E. étaient réhumectés toutes les semaines à l'aide d'un pulvérisateur. Connaissant le poids total (boîte + mélange à $120 \%$ H. E) des incubations, il suffisait par une pesée hebdomadaire d'ajouter la perte de poids en eau distillée. Les variations ainsi enregistrées étaient entre $120 \%$ et $110 \%$ de l'humidité équivalente.

Les échantillons subissant l'hygropériodisme étaient desséchés à l'air et ramenés à $120 \%$ H.E. toutes les quatre ou cinq semaines. Ils ont subi ainsi quatre alternances enregistrant des variations de l'ordre de $120 \%$ H. E. à 2 ou $5 \%$ d'humidité par rapport au poids du mélange sec.

Période d'incubation: 121 jours

\section{Déroulement de l'expérience}

Début : la préparation des incubations a eu lieu le 2 décembre 1964 et les boîtes étant fermées, nous avons laissé l'humidité s'homogénéiser pendant une semaine. 
Le 8 décembre, les mélanges ont été humectés à $120 \%$ de leur humidité équivalente (cette dernière ayant été déterminée à la presse membrane) et mis en incubation.

Les 15 décembre 1964, 13 janvier, 18 février et 29 avril 1965, des analyses microbiologiques des mélanges ont été effectuées sur l'une des répétitions et pour chaque régime d'humidité. Ces analyses consistent en un comptage de la microflore totale, de la population fongique et des actinomycètes ainsi que de la microflore minéralisant et précipitant le fer.

Ces analyses se faisant sur une prise de $5 \mathrm{~g}$ de mélange, des corrections ont dû être faites sur le poids total des échantillons utilisés lors des additions d'eau distillée pour ramener à $120 \% \mathrm{H}$. E.

Fin : à 121 jours, l'incubation a été arrêtée, les échantillons étant tamisés à $2 \mathrm{~mm}$ et placés en chambre froide dans l'attente d'analyse du Mn échangeable et facilement réductible. Les dosages sur les incubations à $120 \% \mathrm{H}$. E. étaient terminés après deux semaines et ceux des incubations avec alternance après 1 mois de chambre froide.

\section{Techniques d'analyse}

$1^{0}$ Analyse microbiologique (Résultats présentés dans la thèse)

Echantillon: une des répétitions des traitements.

Prise : $5 \mathrm{~g}$ du mélange humide.

Dilution : la dilution $10^{-1}$ était obtenue en faisant agiter pendant $30 \mathrm{mn}$ la prise de $5 \mathrm{~g}$ dans $47,5 \mathrm{ml}$ d'eau distillée.

\section{Milieux de culture utilisés}

La microflore totale : milieu de Lochead (EDGell 1960).

Les champignons: milieu Opya-Papavizas (PAPAVIZAS 1959).

Les actinomycètes: milieu de Benedict modifié (PorTER 1960).

Les microflores minéralisant et précipitant le fer : milieu de Harder (1919).

Comptage : les comptages avaient lieu à :

- 7 jours pour la microflore totale

- 7 jours pour les champignons

- 14 jours pour les actinomycètes

- 14 jours pour la microflore précipitant et minéralisant le fer.

Résultats : exprimés par gramme de carbone.

$2^{\circ}$ Analyses chimiques des incubations

- Dosage du carbone : méthode ANNE modifiée (Ann. agron. 1963).

- Dosage des acides humiques : Méthode du permanganate N/10 de DuchauFOUR et JACQUIN (Ann. agron. 1963). 
Le fractionnement en acides humiques bruns, gris et intermédiaires a été fait par électrophorèse (résultats présentés dans la thèse).

- Dosage du Mn échangeable :

1) percolation de $200 \mathrm{ml}$ d'eau distillée après macération de $12 \mathrm{~h}$ (lessivage);

2) percolation de $250 \mathrm{ml}$ d'une solution d'acétate d'ammonium $\mathrm{N}$ à $\mathrm{pH}=$ 7 sur 10 g humides (120\% H. E.) ou secs à l'air (hygropériodisme) des mélanges après macération de $12 \mathrm{~h}$. ;

3) détermination du manganèse par la méthode WILDE et VoIGT modifiée.

- Dosage du Mn facilement réductible :

1) percolation de $250 \mathrm{ml}$ d'une solution d'acétate d'ammonium $\mathrm{N}$ avec $0,2 \%$ de chlorhydrate d'hydroxylamine à $\mathrm{pH}=7$ sur $10 \mathrm{~g}$ de mélange humide $(120 \%$ H. E.) ou sec à l'air (hygropériodisme) après macération de $12 \mathrm{~h}$;

2) détermination du manganèse par la méthode WILDE et VoIGT modifiée.

- $\mathrm{pH}$ : les $\mathrm{pH}$ des incubations à $120 \%$ H. E. et sous hygropériodisme ont été mesurés au $\mathrm{pH}$ mètre après macération de $12 \mathrm{~h}$ de $5 \mathrm{~g}$ de mélange dans 10 et $15 \mathrm{ml}$ d'eau distillée respectivement.

\section{$3^{\circ}$ Analyses chimiques des litières}

1) Minéralisation avec $10 \mathrm{ml}$ de $\mathrm{H}_{2} \mathrm{SO}_{4}$ concentré et $5 \mathrm{ml}$ de $\mathrm{H}_{2} \mathrm{O}_{2}$ (répété si nécessaire) de $500 \mathrm{mg}$ de tissu séché à $65^{\circ} \mathrm{C}$ et broyé à $2 \mathrm{~mm}$ pour obtenir le manganèse total.

2) Lessivage de $2 \mathrm{~g}$ de tissu par $200 \mathrm{ml}$ d'eau distillée après macération de $12 \mathrm{~h}$ pour obtenir le manganèse hydrosoluble.

3) Percolation de $250 \mathrm{ml}$ d'une solution à $\mathrm{pH}=7$ d'acétate d'ammonium $\mathrm{N}$ sur $2 \mathrm{~g}$ de tissu séché à $65^{\circ} \mathrm{C}$ après macération de $12 \mathrm{~h}$ afin d'obtenir le $\mathrm{Mn}$ échangeable.

4) Percolation de $250 \mathrm{ml}$ d'une solution à $\mathrm{pH}=7$ d'acétate d'ammonium $\mathrm{N}$ avec $0,2 \%$ de chlorhydrate d'hydroxylamine sur $2 \mathrm{~g}$ de tissu séché à $65^{\circ} \mathrm{C}$ après macération de $12 \mathrm{~h}$ afin d'obtenir le $\mathrm{Mn}$ facilement réductible. C'est le manganèse se trouvant sous forme d'oxyde supérieur $\left(\mathrm{Mn}_{2} \mathrm{O}_{3}\right.$ ou $\left.\mathrm{Mn}_{3} \mathrm{O}_{4}\right)$ ou de complexe organique $\left(\mathrm{Mn}^{3}+\mathrm{CHO}\right)$ et pouvant être déplacé par un réducteur tel que le chlorhydrate d'hydroxylamine. Certains auteurs le considèrent comme une réserve de $\mathrm{Mn}$ pouvant être disponible pour la nutrition des plantes.

5) Détermination du Mn dans les percolats par la méthode de WiLDE et VoigT modifiée.

\section{b) Résultats des analyses chimiques}

Les résultats essentiels sont mentionnés aux tableaux 2, 3 et 4 .

1) L'analyse des humus caractérise très bien les types mull et moder tant par le pourcent de matière organique, le rapport $\mathrm{C} / \mathrm{N}$, les bases échangeables et la capa- 
cité totale d'échange que par la forte teneur en Mn échangeable et facilement réductible du mull comparé au moder.

2) Notons la différence entre les teneurs en Mn échangeable et facilement réductible des humus à l'état humide et sec à l'air.

3) Le tableau 4 nous donne de précieux renseignements quant à la forme du Mn dans les litières (feuilles) utilisées. Si l'on admet qu'une solution normale d'acétate d'ammonium déplace le manganèse échangeable, c'est-à-dire bivalent $\left(\mathrm{Mn}^{++}\right)$, nous constatons que les résultats obtenus par la minéralisation des tissus (feuilles de hêtre et sapin) se comparent à ceux de la percolation d'acétate d'ammonium. Nous pouvons donc croire que le $\mathrm{Mn}$ dans les feuilles de hêtre et sapin est à l'état bivalent.

4) Le lessivage du Mn des litières par une même quantité d'eau distillée (200 ml) est plus grand (1,5 fois plus) pour le sapin que pour le hêtre.

\section{TABLEAU 2}

Analyses des humus tamisés à $2 \mathrm{~mm}$.

Résultats pour $100 \mathrm{~g}$ d'humus séché à l'air

\begin{tabular}{|c|c|c|c|c|c|c|c|c|c|c|c|c|c|c|}
\hline humus & $\mathrm{pH}$ & $\begin{array}{l}\mathrm{C} \\
\%\end{array}$ & $\underset{\%}{\text { M.O. }}$ & $\begin{array}{l}\mathrm{N} \\
\%\end{array}$ & $\mathrm{C} / \mathrm{N}$ & $\begin{array}{c}\mathrm{K} \\
\mathrm{me}\end{array}$ & $\begin{array}{l}\mathrm{Ca} \\
\text { me }\end{array}$ & $\begin{array}{l}\mathrm{Mg} \\
\text { me }\end{array}$ & $\begin{array}{c}\text { P205 } \\
\%\end{array}$ & $\begin{array}{l}\mathrm{Fe} \\
\%\end{array}$ & $\begin{array}{c}\mathrm{T} \\
\mathrm{me}\end{array}$ & $\begin{array}{l}\text { Mn } \\
\text { éch. } \\
\text { me }\end{array}$ & $\begin{array}{l}\text { Mn } \\
\text { f.r. } \\
\text { me }\end{array}$ & $\% \mathrm{H}$ \\
\hline Mull & 4,4 & 3,04 & 5,2 & 0,14 & 21,7 & 0,29 & 0,86 & 0,13 & 0,09 & 0,48 & 10,0 & 0,32 & 4,79 & 1,83 \\
\hline Moder & 3,4 & 11,44 & 22,9 & 0,44 & 26,0 & 0,38 & 0,93 & 0,63 & 0,06 & 0,15 & 22,4 & 0,05 & 0,07 & 4,89 \\
\hline
\end{tabular}

M.O. ................ Matière organique

$\mathrm{T} \ldots \ldots \ldots \ldots \ldots \ldots \ldots \ldots \ldots \ldots$ Capaté d'échange totale

Mn. éch. ............... Manganèse échangeable seul

Mn. f.r. $\ldots \ldots \ldots \ldots \ldots \ldots \ldots$ Manganèse facilement réductible seul

$\% \mathrm{H} \ldots \ldots \ldots \ldots \ldots \ldots \ldots \ldots \ldots \ldots$ Pourcentage d'humidité

TABLEAU 3

Analyse du Mn échangeable et facilement réductible sur les humus humides tamisés à $2 \mathrm{~mm}$.

Résultats pour $100 \mathrm{~g}$ d'humus séché à $105^{\circ} \mathrm{C}$

\begin{tabular}{|c|c|c|c|}
\hline Humus & Mn éch. & Mn f.r. & $\% \mathrm{H}$ \\
\hline $\begin{array}{l}\text { Mull } \ldots \ldots \ldots \ldots \ldots \ldots \\
\text { Moder } \ldots \ldots \ldots \ldots \ldots\end{array}$ & $\begin{array}{l}0,23 \\
0,086\end{array}$ & $\begin{array}{l}5,35 \\
0\end{array}$ & $\begin{array}{l}22,58 \\
41,92\end{array}$ \\
\hline
\end{tabular}




\section{TABLEAU 4}

Manganèse dans les litières

exprimé en milliéquivalent par $g$ de litière séchée à $65^{\circ} \mathrm{C}$

\begin{tabular}{|c|c|c|}
\hline Litières & Hêtres & Sapins \\
\hline Minéralisation $\ldots \ldots \ldots \ldots \ldots \ldots \ldots$ & 0,179 & 0,151 \\
\hline $\begin{array}{r}\text { Percolation d'acétate d'ammonium } \mathrm{N} \\
\mathrm{pH}=7.0 \ldots \ldots \ldots \ldots \ldots \ldots \ldots\end{array}$ & 0,173 & 0,149 \\
\hline $\begin{aligned} \text { Percolation d'acétate d'ammonium } & \mathrm{N} \\
+0,2 \% \text { de chlorhydrate à } \mathrm{pH}=7 & . .\end{aligned}$ & 0,168 & 0,150 \\
\hline Lessivage de Mn par $200 \mathrm{ml}$ d'eau ..... & 0,076 & 0,093 \\
\hline
\end{tabular}

\section{B. - BILAN PARTIEl DU CyCle du MANGANÈSE} DANS LES TYPES DE SAPINIÈRE A MULL ET MODER

\section{a) Bilan de l'évolution du Mn dans les incubations}

Le tableau 5 donne les teneurs des humus en différentes formes de manganèse après traitement et incubation.

\section{TABLEAU 5}

Mn échangeable et facilement réductible après 19 semaines des traitements, Comparaison avec le point de départ. Résultats en me/100 g séché à $105^{\circ} \mathrm{C}$, Moyennes des répétitions

\begin{tabular}{|c|c|c|c|c|c|c|}
\hline \multirow{3}{*}{ Traitements } & \multirow{2}{*}{\multicolumn{2}{|c|}{ Au début }} & \multicolumn{4}{|c|}{ Après 19 semaines } \\
\hline & & & \multicolumn{2}{|c|}{$120 \%$ H. E. } & \multicolumn{2}{|c|}{ Hygropériodisme } \\
\hline & $\begin{array}{c}\text { Mn éch. (1) } \\
\text { me }\end{array}$ & $\begin{array}{c}\text { Mn f.r. } \\
\text { me }\end{array}$ & $\begin{array}{l}\text { Mn éch. } \\
\text { me }\end{array}$ & $\begin{array}{l}\text { Mn f.r. } \\
\text { me }\end{array}$ & $\begin{array}{l}\text { Mn éch. } \\
\text { me }\end{array}$ & $\begin{array}{l}\text { Mn f.r. } \\
\text { me }\end{array}$ \\
\hline Moder+lit de sapin .... & 4,12 & 0 & 3,659 & 0,037 & 3,052 & 0,155 \\
\hline Moder+lit de hêtre .... & 4,87 & 0 & 0,792 & 4,100 & 1,306 & 2,988 \\
\hline Moder+ $\mathrm{MnSO}_{4} \quad \ldots \ldots$ & 10,39 & 0 & 7,966 & & 6,764 & 0,100 \\
\hline $\begin{array}{l}\text { Moder }+\mathrm{MnO}_{2}\left(\mathrm{Mn}^{4+}=\right. \\
10.06 \mathrm{me})\end{array}$ & & & & & & \\
\hline $\begin{array}{r}10.06 \text { me) } \ldots \ldots \ldots \ldots \\
\text { Moder (témoin) } \ldots \ldots \ldots\end{array}$ & 0,08 & 0 & 3,419 & 3,454 & 1,226 & 3,443 \\
\hline Moder (témoin) . & 0,08 & 0 & 0,106 & & 0,054 & \\
\hline Mull+lit de sapin $\ldots$. & 2,28 & 4,61 & 0,420 & 5,673 & 1,296 & 4,462 \\
\hline Mull+ lit de hêtre ...... & 2,67 & 4,61 & 0,269 & 6,619 & 0,636 & 6,084 \\
\hline $\mathrm{Mull}+\mathrm{MnSO}_{4} \quad \ldots \ldots \ldots$ & 4,75 & 5,33 & 3,151 & 5,375 & 4,904 & 2,952 \\
\hline Mull+ $\mathrm{MnO}_{2}\left(\mathrm{Mn}^{4+}=\right.$ & & & & & & \\
\hline $440 \mathrm{me}) \ldots \ldots \ldots \ldots$ & 0,23 & 5,33 & 0,587 & 8,394 & 1,617 & 6,918 \\
\hline Mull (témoin) . . . . . . . & 0,23 & 5,33 & 0,475 & 4,929 & 1,741 & 3,351 \\
\hline
\end{tabular}

(1) $\mathrm{Mn}$ éch., début $=M n$ éch. add. + Mn éch. Humus. 
Du tableau 5, nous pouvons déduire immédiatement qu'après incubation à $120 \%$ de l'humidité équivalente, la teneur en Mn facilement réductible est généralement beaucoup plus forte dans les mull que dans les moder, quels que soient les traitements appliqués.

Cependant, une analyse plus poussée des résultats est encore plus explicite.

Nous pouvons, pour chaque ligne du tableau, établir un bilan de transformation du manganèse. Par exemple, pour le mull témoin :

$$
\begin{gathered}
0,23 \text { éch. }+5,33 \text { f.r. } \rightarrow 0,475 \text { éch. }+4,929 \text { f.r. }+0,156 \text { n. e. } \\
\text { début } \\
\text { fin de l'incubation }
\end{gathered}
$$

éch. désignant le $\mathrm{Mn}$ échangeable, f.r. le $\mathrm{Mn}$ facilement réductible, n.e. le Mn non extractible.

Est considéré comme manganèse non extractible dans un tel bilan, la quantité de Mn qu'on ne retrouve en fin d'incubation ni sous forme de Mn échangeable, ni sous forme de $\mathrm{Mn}$ facilement réductible : il peut s'agir de manganèse fortement oxydé $\left(\mathrm{Mn}^{4+}\right)$ ou de manganèse complexé dans la matière organique.

Pour le moder, le bilan serait :

$$
0,08 \text { éch. }+0 \text { f.r. } \rightarrow 0,106 \text { éch. }+0 \text { f.r. }-0,026 \text { n.e. }
$$

Ce qui signifie qu'une partie du manganèse non extractible en début d'incubation est devenu échangeable à la fin.

En soustrayant des chiffres de chacune des lignes correspondant aux différents traitements, les chiffres correspondants pour les humus témoins, nous obtenons un bilan du manganèse ajouté dans chaque traitement soit sous forme de litière, soit sous forme de sel ou oxyde $\left(\mathrm{MnSO}_{4}\right.$ ou $\left.\mathrm{MnO}_{2}\right)$. Suivant les cas, ce $\mathrm{Mn}$ ajouté est soit bivalent (litières et $\left.\mathrm{MnSO}_{4}\right)$, soit tétravalent $\left(\mathrm{MnO}_{2}\right)$.

Bien entendu, dans le cas des mélanges litière-humus, on doit corriger le bilan de 1'humus-témoin, à soustraire des chiffres obtenus avec le mélange, au prorata de la quantité réelle d'humus contenue dans le mélange. Le bilan d'humus-témoin devient ainsi :

- pour le mull

$$
0,20 \text { éch. }+4,61 \text { f.r. } \rightarrow 0,42 \text { éch. }+4,26 \text { f.r. }+0,13 \text { n.e. }
$$

- pour le moder

$$
0,07 \text { éch. }+0 \text { f.r. } \rightarrow 0,09 \text { éch. }+0 \text { f.r. }-0,02 \text { n.e. }
$$

On arrive ainsi à dresser un bilan complet qui est représenté en détail au tableau 6 pour les humus incubés en régime d'humidité permanente. Dans ce tableau, le bilan est indiqué à la fois en valeurs absolues et en pourcentage et on a mentionné également le ( rendement) de la transformation du manganèse ajouté (oxydation pour $\mathrm{Mn}$ des litières et $\mathrm{MnSO}_{4}$ et réduction pour $\mathrm{MnO}_{2}$ ). 


\section{TABLEAU 6}

Bilan d'évolution du Mn additionné aux humus sous forme de litière, $\mathrm{MnSO}_{4}$ et $\mathrm{MnO}_{2}$. Rendement de transformation du Mn. Traitement à $120 \%$ humidité équivalente

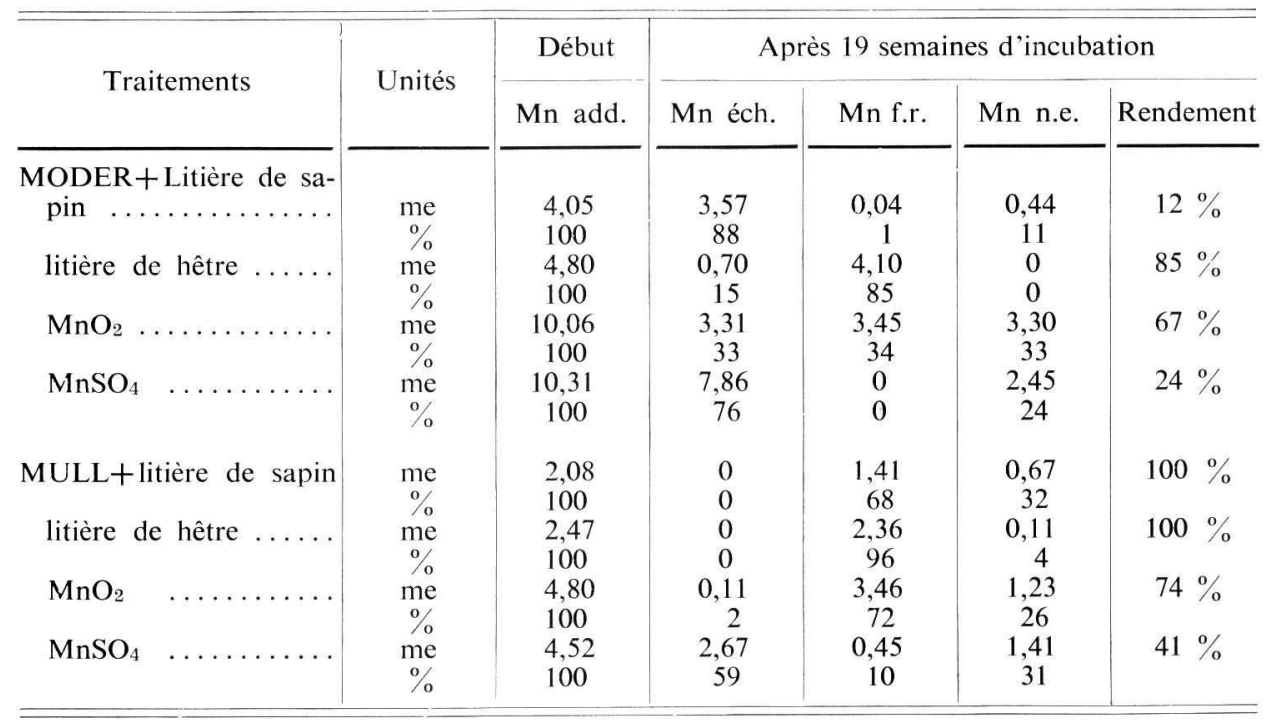

Mn n.e. $=$ Mn non extractible.

Nous pouvons tirer de ce tableau les renseignements suivants :

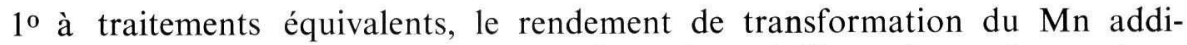
tionné est plus grand pour le mull que pour le moder. D'ailleurs, les analyses microbiologiques nous ont démontré que le mull est beaucoup plus actif que le moder et que par conséquent, les pouvoirs réducteurs et surtout oxydants de la microflore du mull sont supérieurs à ceux du moder. Il faut noter dans le cas des traitements $\mathrm{MnSO}_{4}$ et $\mathrm{MnO}_{2}$, le rôle possible des acides humiques et de l'acidité des humus vis-à-vis de l'oxydation ou de la réduction du Mn. Mais malgré l'acidité du mull, on constate que son pouvoir oxydant sur le $\mathrm{Mn}^{++}$par rapport à celui du moder est deux fois plus grand dans le cas du traitement $\mathrm{MnSO}_{4}$ et dix fois plus dans le cas de la litière de sapin, tandis que le pouvoir réducteur du mull est légèrement supérieur à celui du moder ;

$2^{\circ}$ la transformation du Mn additionné en Mn f.r. est plus forte dans le cas du mulı à traitements équivalents. De plus, si l'on considère que les peuplements de la sapinière vosgienne sont surtout composés de sapin et en particulier sur les stations à moder, on peut admettre ainsi deux évolutions totalement différentes du Mn des litières suivant les stations : sur les stations à mull, le Mn des litières devient à $70 \%$ du Mn f.r. tandis que sur les stations à moder (et probablement à mor), $90 \%$ du Mn des litières restent sous forme échangeable. 
$3^{\circ}$ La litière de hêtre comme le $\mathrm{MnO}_{2}$ donne au moder un comportement semblable au mull même si les rendements sont inférieurs. Nous avons pu faire la même constatation sur l'évolution de la microflore dans le cas du moder-hêtre.

$4^{0}$ Rôle de 1'hygropériodisme: en comparant dans le tableau 5 les résultats obtenus en humidité permanente et en hygropériodisme, nous pouvons conclure que dans le cas du mull, les alternances d'humidité et de sécheresse se traduisent par une diminution du $\mathrm{Mn}$ facilement réductible obtenu en fin d'incubation et par une augmentation du manganèse échangeable ; toutefois, l'augmentation de cette dernière forme de $\mathrm{Mn}$ ne compense pas la diminution constatée du Mn réductible et on doit en conclure qu'une partie de ce dernier est devenue du manganèse non extractible probablement $\mathrm{Mn}^{4+}$.

Dans le cas du moder, au contraire, on constate une diminution du Mn échangeable au profit du Mn non extractible sauf dans le cas d'adjonction de litière de hêtre qui confère au moder un comportement de mull comme dans le cas de l'incubation à humidité constante.

Un bilan de ces diverses transformations dues à l'hygropériodisme est donné au tableau 7.

\section{TABLEAU 7}

Transformation du Mn échangeable ou facilement réductible sous l'influence de l'hygropériodisme

\begin{tabular}{|c|c|c|}
\hline 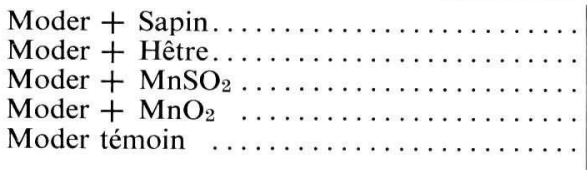 & $\begin{array}{l}0,61 \text { éch. } \\
1,11 \text { f.r. } \\
1,21 \text { éch. } \\
2,19 \text { éch. } \\
0,05 \text { éch. }\end{array}$ & $\begin{array}{l}\longrightarrow 0,12 \text { f.r. }+0,49 \text { n.e. } \\
\longrightarrow 0,52 \text { éch. }+0,31 \text { n.e. } \\
\longrightarrow 0,10 \text { f.r. }+1,1 \text { n.e. } \\
\longrightarrow 0 \text { f.r. }+2,19 \text { n.e. } \\
\longrightarrow 0 \text { f.r. }+0,05 \text { n.e. }\end{array}$ \\
\hline 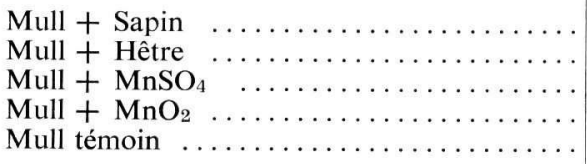 & $\begin{array}{l}1,21 \text { f.r. } \\
0,54 \text { f.r. } \\
2,43 \text { f.r. } \\
1,47 \text { f.r. } \\
1,58 \text { f.r. }\end{array}$ & $\begin{array}{l}\longrightarrow 0,88 \text { éch. }+0,31 \text { n.e. } \\
\longrightarrow 0,37 \text { éch. }+0,17 \text { n.e. } \\
\longrightarrow 1,75 \text { éch. }+0,68 \text { n.e. } \\
\longrightarrow 1,03 \text { éch. }+0,44 \text { n.e. } \\
\longrightarrow 1,26 \text { éch. }+0,22 \text { n.e. }\end{array}$ \\
\hline
\end{tabular}

éch. échangeable, f.r. facilement réductible, n.e. non extractible.

b) Bilan partiel du cycle du manganèse dans les sapinières à mull et moder

Faute de données sur les retombées de litières dans la sapinière, nous avons utilisé des données de la pessière établie par H. MaYer Krapoll en Allemagne. Nous avons assimilé à la sapinière les fertilités de la pessière de la façon suivante :

— fertilité de la pessière avec $4150 \mathrm{~kg} / \mathrm{ha} / \mathrm{an}$ de retombées correspond à la sapinière à mull dont la composition est de $75 \%$ de sapin et $25 \%$ de hêtre ;

- fertilité de la pessière avec $2775 \mathrm{~kg} / \mathrm{ha} / \mathrm{an}$ de retombées correspond à la sapinière à moder dont la composition est de $95 \%$ de sapin et $5 \%$ de hêtre. 
Pour évaluer la contribution du hêtre dans les retombées annuelles, nous avons appliqué le pourcent de hêtre dans le peuplement aux retombées d'une hêtraie pure soit $4440 \mathrm{~kg} / \mathrm{ha} / \mathrm{an}$, chiffre établi par l'auteur précité.

Le bilan a été fait pour deux roches-mères et deux types d'humus : mull et moder. Des analyses foliaires de sapin et de hêtre suivant les humus ont permis finalement de calculer les retombées annuelles totales possibles de Mn. En appliquant les proportions de transformation du Mn trouvé dans les incubations aux retombées en $\mathrm{Mn}$ en respect de chacune des espèces (sapin et hêtre), nous pouvons évaluer l'ordre de grandeur de l'enrichissement en Mn facilement réductible des mull et moder de sapinière (tableau 8).

TABLEAU 8

Retombées en Mn et enrichissements possibles en humus en Mn f.r. et Mn éch.

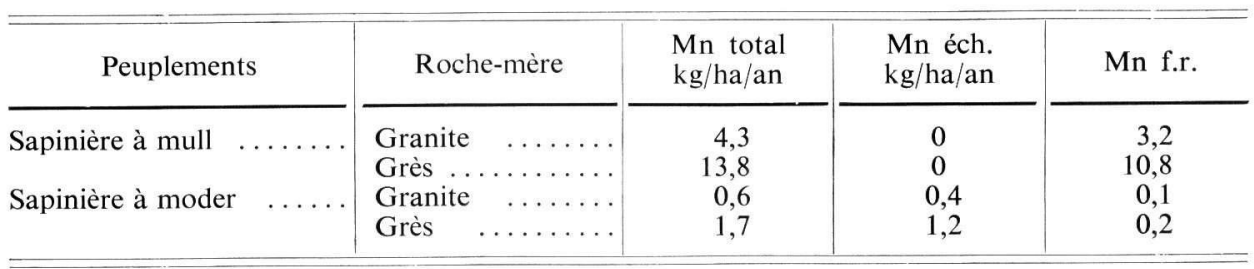

Les feuilles de hêtre poussant sur station à mull sur granite et grès ont respectivement $0,102 \%$ et $0,452 \%$ de $\mathrm{Mn}$ et sur station à moder $0,072 \%$ et $0,121 \%$, tandis que pour le sapin, on trouve $0,101 \%$ et $0,283 \%$ dans le cas du mull sur rochemère granitique et gréseuse contre $0,017 \%$ et $0,053 \%$ dans le cas de stations à moder.

\section{Commentaires}

Ces résultats quoiqu'approximatifs nous donnent une idée des apports en Mn des litières suivant le type d'humus et de roche-mère et, par conséquent, l'enrichissement possible en Mn f.r.

Il est extraordinaire de constater une si forte différence entre la sapinière à mull et à moder du point de vue de l'apport en Mn total et de l'enrichissement possible en $\mathrm{Mn}$ f.r. Cela explique les quantités importantes de $\mathrm{Mn}$ facilement réductible dosé dans les mull de sapinière allant jusqu'à 9,57 me pour $100 \mathrm{~g}$ ou 2600 ppm tandis que l'on trouve de 0,07 à 1,46 dans le moder de sapinière et de hêtraie (tableau 9).

\section{Interprétation des résultats}

Le nombre de relevés au tableau 9 ne nous permet pas de nous prononcer sur la relation entre la régénération de sapin et la concentration en Mn échangeable et Mn facilement réductible, d'autant plus que le phénomène de toxicité est de nature physico-chimique. 


\section{TABLEAU 9}

Comparaison des quantités de Mn éch. et Mn f.r. dans les humus de sapinière et hêtraie. Milliéquivalents pour $100 \mathrm{~g}$ séchés à l'air

\begin{tabular}{|c|c|c|c|c|}
\hline \multirow{2}{*}{ Peuplements } & \multirow{2}{*}{$\begin{array}{l}\text { Régéné- } \\
\text { ration }\end{array}$} & \multirow{2}{*}{ Roches-mères } & \multicolumn{2}{|c|}{ Horizon A } \\
\hline & & & $\begin{array}{l}\text { Mn éch. } \\
\text { me/100 g }\end{array}$ & $\begin{array}{l}\text { Mn f.r. } \\
\mathrm{me} / 100 \mathrm{~g}\end{array}$ \\
\hline $\begin{array}{l}\text { Sapinière à hêtre (mull) } \\
\text { Forêt de Mortagne P-51 ....... }\end{array}$ & - & Grès intermédiaire & 0,32 & 9,57 \\
\hline $\begin{array}{l}\text { Sapinière à hêtre (mull) } \\
\text { Forêt de Mortagne P-49 ....... }\end{array}$ & - & Grès intermédiaire & 0,42 & 3,92 \\
\hline $\begin{array}{l}\text { Sapinière à hêtre (mull) } \\
\text { Ft du Ban d'Etival P-3 }\end{array}$ & Hêtre & Grès intermédiaire & 0,32 & 4,72 \\
\hline $\begin{array}{l}\text { Sapinière à hêtre (mull) } \\
\text { Forêt de Mortagne P-49. }\end{array}$ & Sapin & Grès intermédiaire & 0,24 & 5,20 \\
\hline $\begin{array}{l}\text { Sapinière à hêtre (mull) } \\
\text { Forêt de Mortagne P-51 . }\end{array}$ & Sapin & Grès intermédiaire & 0,26 & 2,95 \\
\hline $\begin{array}{l}\text { Sapinière à hêtre (mull) } \\
\text { Ft du Ban d'Etival P-2 } \\
\text { Sapinière à hêtre (mull) }\end{array}$ & $\begin{array}{l}\text { Sapin } \\
\text { Hêtre } \\
\text { Sanin }\end{array}$ & Grès intermédiaire & 0,72 & 4,88 \\
\hline $\begin{array}{l}\text { Forêt de Badonviller ...... } \\
\text { Sapinière à hêtre (mull) }\end{array}$ & Hêtre & Grès intermédiaire & 0,14 & 3,30 \\
\hline $\begin{array}{l}\text { Ft de Malmerspach } \ldots \ldots \ldots \\
\text { Sapinière à mull-moder } \\
\text { Ft de Malmerspach } \ldots \ldots \ldots\end{array}$ & $\begin{array}{l}\text { Hêtre } \\
\text { Sapin } \\
\text { Hêtre }\end{array}$ & Grauwacke du Dévonien & 0,13 & 4,89 \\
\hline $\begin{array}{l}\text { Sapinière à hêtre (mull) } \\
\text { Forêt de Saint-Quirin }\end{array}$ & Hêtre & Grès intermédiaire & 0,06 & 2,94 \\
\hline $\begin{array}{l}\text { Sapinière de moder } \\
\text { Forêt de Saint-Quirin . }\end{array}$ & Sapin & Conglomérat & 0,44 & 0,65 \\
\hline $\begin{array}{l}\text { Sapinière à moder } \\
\text { Forêt du Ban d'Etival } \\
\text { Hêtraie à Chêne }\end{array}$ & Sapin & Conglomérat & 0,05 & 0,07 \\
\hline $\begin{array}{l}\text { Forêt de Badonviller } \ldots \\
\text { Hêtraie à Chêne }\end{array}$ & Sapin & Grès intermédiaire & 0,00 & 0,59 \\
\hline $\begin{array}{l}\text { Forêt de Badonviller } \\
\text { Hêtraie à Chêne }\end{array}$ & Sapin & Grès vosgien & 0,08 & 0,46 \\
\hline Forêt de Badonviller & Sapin & Grès intermédiaire & 0,11 & 0,39 \\
\hline
\end{tabular}

Cependant, il est reconnu que les difficultés de régénération du sapin sont liées au type d'humus mull qui, en règle générale, est riche en $\mathrm{Mn}$ facilement réductible. Au contraire, le Sapin se régénère très bien sur le moder qui contient peu ou pas de Mn facilement réductible. Mais le tableau 9 nous montre qu'il existe des cas où une régénération a pu s'installer sur des mull bien qu'ils soient riches en $\mathrm{Mn}$ réductible.

Donc, nous pouvons formuler l'hypothèse que le $\mathrm{Mn}$ facilement réductible n'est pas directement toxique mais qu'en période de sécheresse, lorsqu'il a atteint une certaine concentration, il serait à l'origine de la toxicité manganique en se dismutant. D'ailleurs Rousseau (1960) a montré qu'une phase de dessiccation était nécessaire pour que s'y manifeste la toxicité manganique.

Rappelons à ce sujet, les résultats de nos incubations ayant subi l'hygropériodisme. 
Nous avons vu que l'alternance de sécheresse et d'humidité provoquait une dismutation du Mn f.r. au profit du Mn éch. Par exemple, quatre alternances de ce genre ont élevé la concentration en $\mathrm{Mn}$ éch. à 1,296, 0,636 et 1,741 me pour $100 \mathrm{~g}$ de mélange des traitements mull + hêtre, mull + sapin et mull + témoin (tableau 5), ce qui fait 356, 175 et $478 \mathrm{ppm}$. Ces chiffres sont supérieurs à la plus forte concentration en hydroponique utilisée par l'expérience de RoussEAU (1960) : $110 \mathrm{ppm}$. Donc la concentration du Mn éch. est dépendante de la concentration en Mn f.r. et indirectement des variations d'humidité de l'humus, ce dernier facteur dépendant du climat.

Par ce fait, il est très possible que, malgré une forte concentration en Mn f.r., une régénération en sapin puisse s'accomplir à condition que les trois ou quatre saisons estivales qui suivront une germination ne soient pas marquées par de fortes sécheresses, ce qui expliquerait la présence d'une régénération sur des humus mull pouvant être toxiques par leur réserve en $\mathrm{Mn}$ f.r.

Les germinations qui seraient suivies d'un été à période sèche seraient condamnées car la dismutation du Mn f.r. provoquerait une intoxication manganique.

Il a été observé qu'une régénération âgée de 4 ou 5 ans ne pouvait être détruite et avait passé le cap de survie car son enracinement avait atteint la base de l'horizon $\mathrm{A}_{1}$ et l'horizon B. L'objection de la présence de forte concentration en Mn f.r. dans l'horizon B ne prévaut pas sur le plan toxicité car cet horizon, comme la base de l'horizon $\left(A_{1}\right)$, ne subit jamais de dessèchement total et le Mn éch. est à l'état de traces dans les horizons $\mathrm{B}$.

L'intoxication des semis de sapin sur les mull doit donc être considérée comme le résultat de la conjugaison d'un facteur microbiologique permanent et un facteur climatique occasionnel.

Le facteur microbiologique est responsable de l'accumulation du Mn f.r. dans les mull de sapinière à hêtre. Comment s'accomplit cette accumulation ? Par le jeu de la roche-mère et de la microbiologie du mull.

La roche-mère, suivant qu'elle est plus ou moins riche en $\mathrm{Mn}$, favorise une assimilation par les plantes qui retournent celui-ci à l'humus par la litière.

La microflore du mull transforme le $\mathrm{Mn}$ des litières en $\mathrm{Mn}$ facilement réductible qui s'accumule dans l'humus jusqu'à atteindre une concentration telle qu'une seule dismutation peut accroître très fortement le taux de Mn échangeable dans l'humus et être toxique aux semis de sapin.

\section{CONCLUSION D'ENSEMBLE}

Les résultats précédents permettent de donner une explication cohérente des difficultés de régénération du sapin dans les Vosges.

Rappelons que c'est surtout dans les stations à mull que la survie du jeune semis est difficile et que Rousseau a pu mettre en évidence dans ce cas une intoxication manganique.

Dans les stations à mull, les aiguilles sont beaucoup plus riches en Mn que sur les moder. Il y a donc apport au sol par la litière de quantités beaucoup plus impor- 
tantes de cet élément dans le premier cas que dans le second. Cet apport se fait dans les deux cas sous forme de manganèse bivalent.

De plus, la microflore agit de manière très différente dans les deux humus. Celle du mull oxyde rapidement une forte proportion du manganèse échangeable de la litière en manganèse facilement réductible qui s'accumule dans l'humus. Sous l'influence de celle du moder, au contraire, il reste en majeure partie sous forme bivalente, échangeable et peut être lessivé par les eaux de pluie.

On constate d'ailleurs que les mull sont beaucoup plus riches que les moder en manganèse facilement réductible, jusqu'à 100 fois plus dans certains cas. Cela correspond d'ailleurs bien au bilan théorique d'évolution du manganèse que l'on peut effectuer à partir de nos essais en incubation.

Ce manganèse facilement réductible étant peu soluble n'est pas toxique par lui-même bien que certains auteurs le croient assimilable. Il ne le devient qu'en libérant par dismutation, pendant les fortes sécheresses de l'été, des quantités importantes de manganèse échangeable qui est seul assimilable.

Cette dismutation, déjà connue, a pu être montrée à nouveau dans notre travail en faisant subir à nos échantillons en incubation des alternances de sécheresse et d'humidité.

Les résultats de nos recherches expérimentales coïncident ainsi très bien avec les conclusions données par Rousseau (1960) sur le même sujet et avec les observations de terrain : il n'y a de toxicité manganique que sur les mull et à la faveur d'un été sec. Cela explique que des régénérations convenables de sapin puissent se produire, même sur mull, si elles ont eu la chance de bénéficier de trois ou quatre étés constamment humides leur permettant d'échapper à cette période critique pendant laquelle les racines sont dans l'horizon superficiel.

Rappelons également que cette dismutation du manganèse réductible qui est à l'origine de la toxicité voit son rôle encore accru par le fait que, comme l'a montré RousSEAU, les mull ont tendance à se dessécher beaucoup plus que les moder pendant les périodes chaudes.

Enfin, l'étude in vitro de l'évolution du manganèse de la litière de hêtre lorsqu'elle est mélangée à un moder de sapin nous a montré que ce manganèse échangeable était abondamment transformé en manganèse réductible comme c'était le cas lorsque nous ajoutions de la litière de sapin à un mull de sapin.

Autrement dit, le moder de sapinière s'il reçoit de la litière de hêtre tend à se transformer sur le plan de l'action de la microflore, en mull.

Ainsi se trouve expliquée l'action sur la régénération des mélanges d'essences et on peut présenter le schéma théorique suivant :

- bonne dans une sapinière pure à moder, la régénération devient médiocre si cette sapinière est envahie par le hêtre car alors le moder se transforme progressivement en mull et s'enrichit en manganèse réductible qui peut devenir toxique ;

- au contraire, sous une hêtraie pure, l'humus reste un moder et l'installation d'un semis de sapin y devient possible. Beaucoup de vieilles hêtraies pures sont ainsi facilement colonisées par le sapin.

Nous arrivons donc ainsi à une explication schématique assez satisfaisante du phénomène (c d'alternance d'essences ) (alternance du sapin et des feuillus) souvent 
constaté dans les Vosges. Elle est liée à l'évolution de l'humus de la forêt mélangée vers un mull où le manganèse s'accumulant sous forme réductible peut devenir toxique. La proportion de sapin diminue alors et la forêt tend vers la hêtraie pure. La régénération du sapin ne peut se maintenir que si la sylviculture permet le maintien d'un humus partiellement dégradé, le moder.

\section{Reçu pour publication en novembre 1967}

\section{SUMMARY}

THE ROLE OF MANGANESE IN FIR STANDS REGENERATION IN VOSGES

Foliar analysis have shown that needles of firs growing on mull soils in Vosges are much richer in manganese than needles of trees growing on mor or moder soils.

After the fall, in needles the manganese is in divalent form and so it is exchangeable.

Incubation has shown that in mull, divalent manganese is quickly oxidized and accumulated in humus. On the other hand, in moder and mor, it remains in the divalent state and may be leached out. Thus, under natural conditions, mull is much richer in oxidized manganese than moder. During summer drying-up, the reduction of this manganese yields large quantities of soluble manganese that may be toxic to the seeds.

\section{ZUAMMENFASSUNG}

BEDEUTUNG DES MANGANS Für DIE VERJUNGUNG DES TANNENBESTANDES IN DEN VOGESEN

An Hand von Nadelanalysen konnte festgestellt werden, dass der Mangangehalt der Nadeln von Tannen auf Mullstandorten in den Vogesen wesentlich höher ist als auf Moder oder Rohhumusstandorten.

In den abgefallenen Nadeln liegt das Mangan als zweiwertiges Kation vor und kann in seiner austauschbaren Form extrahiert werden.

Humusinkubationen haben gezeigt, dass im Mull das zweiwertige Mangan sich sehr rasch in reduzierbares Mangan umformt und sich im Humus anhäuft, während es im Moder und Rohhumus (Mor) als zweiwertiges Kation erhalten bleibt und ausgewaschen werden kann.

Es konnte festgestellt werden, dass Mull unter natürlichen Bedingungen wesentlich mehr reduzierbares Mangan enthält als Moder. Während der sommerlichen Trockenperioden verformt sich das reduzierbare Mangan in bedeutende Mengen an zweiwertigen Mangan, welches auf die Sämlinge toxisch wirken kann.

\section{RÉFÉRENCES BIBLIOGRAPHIQUES}

Alexander M., 1961. - Indroduction to soil microbiology. John Wiley and sons Inc, New York and London.

Amito L.P., Bernier B., 1962. - Méthodes d'analyses chimiques usuelles des sols et des tissus végétaux. 117 p., Les Presses de l'Université, Laval.

Boquel G., Kauffman J., 1963. - Influence du thermopériodisme et de l'hygropériodisme sur la formation de l'humus et l'activité des fixateurs d'azote aérobies libres dans la terre. Cahiers O.R.S.T.O.M. Pédologie, $\mathrm{n}^{\circ}$ 2, 5-7.

Bonner J., Galaton A.W., 1952. - Principle of plan Physiology. W.H. Freeman and Cie. San Francisco.

Duchaufour Ph., 1965. - Précis de Pédologie. Masson et Cie. Paris.

Duchaufour Ph., Parde J., Jacamon M., Debazac E.F., 1958. - Un exemple d'utilisation pratique de la cartographie des stations : la forêt du Ban d'Etival (Vosges). Extrait de la R.F.F., Nancy.

Duchaufour Ph., Jacquin F., 1963. - Recherche d'une méthode d'extraction et de fractionnement des composés humiques par électrophorèse. Ann. agron., 14, (6), 885-918. 
Egdell J.W. et coll., 1960. - Some studies of the colony count technique for soil bacteria. J. appl. Bacteriol., 23, I, 69-86.

GAREY, BARBER, 1952. - Evaluation of certains factors involved in increasing manganese availability with sulfur. Soil Sci. Soc. Amer. Proc., 16, 173-175.

Gerretsen F.C., 1937. - Manganese deficiency of oats and its relation to soil bacteria. Ann. Bot., 1, 207-230.

Harder, 1919. - U.S. Geology survey professional. Papers, n⿳0 113.

JoNes L.N.R., 1957. - The effect of liming a neutral soil on the cycle of manganese. Plant. and soil, 3, 4, 315-327.

La Gunas G.R., 1964. - Relationship between the Fe, Cu, Mn and Mo content of plants and the quantities present in and the $\mathrm{pH}$ of the soil and species of Grenium Quercus. Ann. Edapol. Agrotiol., 23, (1/2), 91-7.

Lukashev K.I., Petukhova N.N., 1963. - The migration and form of accumulation of manganese in soil and plant of the south eastern forest. Dokl. akad. Nauk, SSSR 6, 251-254. Ref. bibliogr. Soils and fertilizers, 26 (2), p. 86, 589.

Man P.J.G., Quastel J.H., 1946. - Manganese metabolism in soil. Agriculture research council unit of soil metabolism. Nature, août ,158-154-156.

Mather K., 1965. - Analyse statistique en biologie. Acta Gauthier-Villars, Paris.

Mayer-Krapoll H., 1964. - Die Dungung im Walde. Auswertung Lang jähriger Versuche und Erfahrungen. Dusseldorf.

Mangenot F., JaCQUin F., 1960. — Produits d'humidification des sciures. Quelques caractéristiques physiques et chimiques. Plant and soil, 13, 291-296.

Mulder E.G., Gerretsen F.C., 1952. - Manganese in relation to plant growth. Adv. agron., 4, 22.1-227.

Papavizas G.C., Davey C.B., 1959. - Evaluation of various media and antimicrobial agent for isolation of soil fungi. Soil Sci., 88 (2).

Penkov O.G., 1962. - Manganese and boron in gray cinnamon soils and solonetses in thekarabakh steppe in the Kura-Araks Lowlands. Dokl.s. Kh. Adad. Timiryazevz, n ${ }^{\circ}$ 76, 113-120.

Perriaux J., 1961-1962. — Etude de la série permo-triasique de la feuille de Bruyère au 1/50000. Bulletin du service de la carte géologique de France, 58, 264.

Pochon I., DE BARJAC H., 1958. - Traité de microbiologie des sols. Applications agronomiques. Dunod, Paris.

Perter J.N. et coll., 1960. - Method for the preferential isolation of actinomycetes from soils. Appl. Microbiol., 8, 3, 174-178.

Rao C.R., 1962. - Advanced statistical method in biometric research. John Wiley, New York.

Robighet, 1957. - Recherches sur les oxydes de fer et de manganèse dans les sols. Ann. agron., 4, $511-522$.

Rousseau L., 1960. - De l'influence du type d'humus sur le développement des plantules de sapin dans les Vosges. Ann. Ec. nation. Eaux et Forêts Stn. Rech. expér. forest., 17, 1.

Sanchez, Kamprath, 1959. - Effects of liming and organic matter on the availability of native and applied manganese. Soil Sci. Soc. Amer. Proc., 23, 302-304.

SNedeCor G.W., 1956. - Statistical methods, applied to experiments in agriculture and biology. The Iowa State university press, Ames, Iowa, USA.

Tomassone R., 1965. - L'analyse des composantes principales. C.N.R.F. Stn. Biométr., Note sci. $n^{\mathbf{0}} 1$, Nancy.

Tomassone R., 1965. - L'analyse discriminante progressive : cas de deux populations. C.N.R.F. Stn. Biométr., AG 65006 , Nancy.

Tomassone R., 1966. - L'analyse de variance et de covariance hiérarchique. C.N.R.F. Stn. Biométr., Note sci. $n^{\circ}$ 2, Nancy.

Vavra, Frederik, 1952. - The effect of sulfur oxydation in the availability of manganese. Soil Sci. Soc. Amer. Proc., 16, 141-144.

VEDY J.C., 1965. - Action des litières en décomposition sur l'évolution des éléments minéraux dans les sols. Thèse Faculté des Sciences de Nancy. 